\title{
Role of Donor-Acceptor Strengths and Separation on the Two-Photon Absorption Response of Cytotoxic Dyes: A TD-DFT Study
}

\author{
Ekaterina A. Badaeva and Tatiana V. Timofeeva \\ Department of Natural Sciences, Highlands University, Las Vegas, New Mexico 87701 \\ Artëm Masunov and Sergei Tretiak* \\ Theoretical Division and Center for Nonlinear Studies, Los Alamos National Laboratory, \\ Los Alamos, New Mexico 87545
}

Received: April 25, 2005; In Final Form: June 15, 2005

\begin{abstract}
Time-dependent density functional theory (TD-DFT) is applied to model one-photon (OPA) and two-photon (TPA) absorption spectra in a series of conjugated cytotoxic dyes. Good agreement with available experimental data is found for calculated excitation energies and cross sections. Calculations show that both OPA and TPA spectra in the molecules studied are typically dominated by two strong peaks corresponding to different electronic states. We find that donor-acceptor strengths and conjugated bridge length have a strong impact on the cross-section magnitudes of low- and high-frequency TPA maxima, respectively. These trends are analyzed in terms of the natural transition orbitals of the corresponding electronic states. Observed structureproperty relationships may have useful implications on design of organic conjugated chromophores with tunable two-photon absorption properties for photodynamic therapy applications.
\end{abstract}

\section{Introduction}

Two-photon absorption (TPA) is defined as the electronic excitation of a molecule induced by a simultaneous absorption of a pair of photons of the same or different energy. This phenomenon was first predicted by M. Göppert-Mayer in 1931, but it was not observed until the advent of lasers. The emergence of technologies that can exploit TPA has attracted significant interest in the fields of chemistry, biology, and photonics. This, in turn, inspired a broad quest searching for functional chromophores with enhanced TPA properties. ${ }^{2-13}$

TPA is a third order nonlinear optical process. Its cross section is quadratically proportional to the intensity of the incident light which provides improved spatial selectivity in three dimensions with up to one wavelength resolution. Moreover, TPA is induced at a frequency of half the actual energy gap which stretches the accessible range of conventional lasers (longer wavelengths at $700-1300 \mathrm{~nm}$ ) and ensures deep penetration depth into an absorbing medium. These distinct properties enable a large variety of TPA applications ${ }^{14,15}$ such as fabrication of optoelectronic logical circuits, ${ }^{16}$ high-resolution fluorescence microscopy and characterization, ${ }^{17-21}$ three-dimensional optical data storage, ${ }^{22-25}$ optical power limiting, ${ }^{26,27}$ upconversion lasing, ${ }^{28,29}$ nondestructive imaging of biological tissues, ${ }^{30-34}$ photodynamic therapy, ${ }^{35-37}$ and new nanobiophotonics applications. ${ }^{38,39}$

In particular, photodynamic therapy is a relatively new approach for targeted cellular apoptosis in biological tissues, with current applications in the treatment of tumors, cancers, blood purification and blindness. ${ }^{35,40-42}$ This therapy involves a selective uptake and retention of a photosensitizer by the target area (e.g., tumor) followed by irradiation with light

*Corresponding author. E-mail: serg@cnls.lanl.gov. of a particular wavelength. This is intended to induce tumor apoptosis, presumably through the formation of free radicals and singlet oxygen. A number of photosensitizers that utilize one-photon absorbing mechanism have been described in the literature. Even though TPA based approaches hold a considerable advantage over conventional OPA technique due to spatial resolution and deep penetration of long wavelength irradiation into tissues, only a few organic photosensitizers based on a TPA mechanism have been suggested (e.g., Rodamine B). ${ }^{43}$

Recently researchers started reevaluation of properties and application of already known NLO compounds. ${ }^{6,8,15,44}$ For example, utilizing complex interplay between substitution and branching makes possible design of complex chromophores with synthetically tunable broad variation of nonlinear properties. ${ }^{12,13,45}$ Compounds with high TPA activity are typically described with general formula $\mathrm{D}-\pi-\mathrm{A}-\pi-\mathrm{D}$ or $\mathrm{A}-\pi-\mathrm{D}-$ $\pi-\mathrm{A}$. Several examples of such compounds were found in series of cyclohexanones (Figure 1a). ${ }^{46-50}$ Significant TPA absorbancies of cyclohexanones were reported in ref 51. It is remarkable that series of compounds with similar molecular structure (piperidones, in neutral and protonated form, Figure 1a) was identified as cytotoxic chemotherapeutic agents. ${ }^{52-56}$ Later it was proposed that combination of TPA activity with cytotoxicity made both series of compounds promising for bioimaging and two-photon photodynamic therapy. ${ }^{57-60}$ Structural characteristics of cyclohexanones and piperidones have been described in numerous publications. ${ }^{56,57,61-65}$ Spectral characteristics, including OPA, TPA and fluorescence of some of those compounds, have also been investigated. ${ }^{58-60,66}$ However, despite these extensive experimental studies, theoretical investigations of these molecular structures have not been done yet. Given the technological importance and possible biomedical applications of such materials, there is a clear need to further investigate the photophysical properties of these unique dyes. 

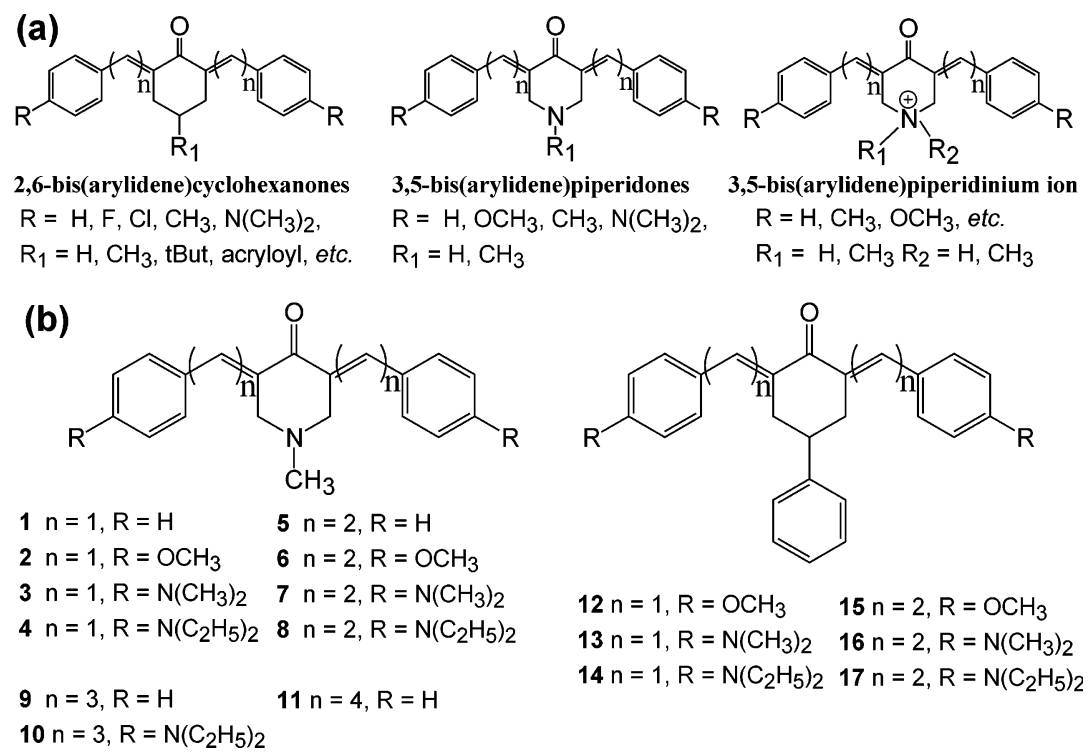

Figure 1. (a) General scheme of arylidenecyclohexanone and arylidenepiperidone derivatives and (b) structures of piperidone (1-11) and cyclohexanone (12-17) chromophores 1-17 studied in this work. Index $n$ denotes the number of vinyl units (i.e., the number of double bonds in the conjugated bridge).

In this article we conduct a computational study of OPA and TPA responses of series of arylidenepiperidone dyes (Figure 1b) as a function of their chemical composition and molecular conformation using time-dependent density functional theory (TD-DFT) in combination with quasi-particle formalism for nonlinear optical polarizabilities. ${ }^{67}$ Details of our computational approach are presented in section 2 . In section 3 , we analyze the computational results and compare them to experiment. Finally, we discuss the trends that emerge and summarize our findings in section 4.

\section{Theoretical Methodology}

Computer design of nonlinear chromophores should allow accurate prediction of stable conformal structures of complex molecules, their fluorescent properties, and nonlinear optical responses and allow the understanding of the underlined structure-property relations, which would ultimately guide organic synthesis. In principle, wave function based correlated $\mathrm{ab}$ initio methods (e.g., equations of motion with a coupledcluster approach (EOM-CC $)^{68}$ ) can provide an accurate description of the electronic spectra. ${ }^{69}$ However, these techniques are frequently computationally intractable, when applied to the molecules of practical interest. Semiempirical methods are numerically feasible, however, they are able to reproduce only certain quantities assumed by underlying parametrization of the Hamiltonian model. ${ }^{70,71}$ The nonlinear spectra are typically dominated by higher excitation levels involving significant electronic correlations. Consequently, semiempirical models have somewhat limited quantitative performance for nonlinear optical responses, while providing an excellent qualitative insight into the nature of physical phenomena involved. ${ }^{6,8}$

Adiabatic TD-DFT ${ }^{72,73}$ in the Kohn-Sham (KS) form is currently the method of choice for calculating the excited-state structure of large molecular systems. ${ }^{74,75}$ Recently TD-DFT extensions for the calculations of molecular nonlinear optical properties have been suggested based on the residues of the quadratic response functions for TPA, ${ }^{76}$ and on the more general quasi-particle formalism of the TD-KS equations for arbitrary frequency-dependent nonlinear optical polarizabilities. ${ }^{77,78} \mathrm{Sub}$ sequently, the latter approach was applied to calculate OPA and TPA responses of several families of donor/acceptor substituted conjugated organic chromophores. ${ }^{79-81}$ These studies have shown an excellent quantitative performance of TD-DFT based on hybrid functionals for both OPA and TPA properties.

In this study, we apply the approach described above ${ }^{78,79}$ to model linear and two photon absorption spectra of chromophores 1-17 shown in Figure 1b. Our calculations start with optimization of ground-state geometries at the Hartree-Fock (HF) level with a 6-31G basis set. We found a good agreement between obtained geometries and available experimental X-ray diffraction data $^{57}$ (see section 3). This is consistent with our previous results $^{79}$ and, subsequently, HF/6-31G optimization level was used across the entire series. TD-DFT formalism as implemented in the Gaussian 98 program package ${ }^{82}$ was then used in combination with the Becke three parameter hybrid exchangecorrelation functional (B3LYP) ${ }^{83,84}$ and 6-31G basis set to calculate excited-state electronic structure. Finally, the modified collective electronic oscillator (CEO) $\operatorname{code}^{67,78}$ was used to calculate OPA and TPA responses based on Gaussian 98 output. A detailed description of our computational technique is given in refs 79 and 80 .

The quantities of interest are first $(\alpha(\omega))$ and third $(\gamma(\omega, \omega,-\omega))$ order frequency-dependent electronic polarizabilities. The OPA intensity at frequency $\omega$ is given by ${ }^{85}$ the imaginary part of

$$
\alpha(\omega)=\sum_{v} \frac{\Omega_{v} \mu_{\mathrm{g} v} \mu_{\mathrm{g} v}^{*}}{\Omega_{v}{ }^{2}-(\omega+i \Gamma)^{2}}
$$

where $\mu_{\mathrm{g} v}$ is the transition dipole moment associated with the electronic transition between the ground $(g)$ and excited $(v)$ states, $\Omega_{\mathrm{g}}$ is the vertical transition frequency, and $\Gamma$ is the line width. We assumed an empirical value $\Gamma=0.1 \mathrm{eV}$ for all OPA and TPA calculations. Similarly, the TPA cross section $\sigma_{\text {TPA }}$ is a function of the imaginary part of $\gamma(\omega, \omega,-\omega): 6,7,15,86,87$

$$
\sigma_{\mathrm{TPA}}(\omega)=\frac{4 \pi^{2} \hbar \omega^{2}}{n^{2} c^{2}} L^{4} \operatorname{Im}\langle\gamma(\omega, \omega,-\omega)\rangle
$$

where $\hbar$ is Planck's constant, $n$ is the refractive index of the medium, $c$ is the speed of light, $L$ is the local field factor 


\begin{tabular}{|c|c|c|c|}
\hline \multicolumn{3}{c|}{ Equatorial } \\
\hline $\begin{array}{c}\text { Side } \\
\text { view }\end{array}$
\end{tabular}

Figure 2. Equatorial and axial conformations of compound 7 (top) and natural transition orbitals describing OPA and TPA states in the axial conformer. The percentage indicates a fraction of the NTO pair contribution to a given electronic excitation.

calculated in the spherical cavity approximation, and

$$
\langle\gamma\rangle=\frac{1}{15}\left(3 \sum_{i} \gamma_{i i i i}+\sum_{i \neq j}\left(\gamma_{i i j j}+\gamma_{i j j i}+\gamma_{i j i j}\right)\right)
$$

is the orientational average of the third-order polarizability tensor $\gamma_{i j k l},{ }^{88}$ where indices $i$ and $j$ refer to the spatial directions $x, y$ and $z$.

The first 12 singlet excited states were taken into account in every calculation. Increasing of the number of the excited states was found to have a negligible impact on UV-visible absorption spectra and only a small effect (within $10 \%$ ) on the magnitude of the TPA cross sections. In the previous study we found 11 states to be sufficient to reach asymptotic values in the resonant third-order responses in linear substituted conjugated chromophores. ${ }^{80}$ To evaluate solvent effects we use the polarizable continuum model (PCM) based on the integral equation formalism (IEF) ${ }^{89-92}$ as implemented in Gaussian $98 .{ }^{82}$ Electronic structures have been calculated for selected compounds in the presence of chloroform (medium polarity solvent with $\epsilon=4.81$ ). Solvent-induced trends (red-shifts of the electronic transition energies and enhancements of TPA cross sections; see section 3) remain the same for any other variation of PCM.

Finally, to understand the nature of the excited states involved into linear and nonlinear absorption processes, we utilize a natural transition orbital (NTO) analysis. ${ }^{93}$ This approach provides the most compact representation of the electronic transition in terms of an expansion into single particle orbitals by diagonalizing the transition density matrix associated with each excitation. In this study, each excited state in question could be well represented as a transition between a single pair of orbitals with contribution of about $80 \%$ or more to the total transition. Figures showing natural transition orbitals were obtained using XCrysDen graphic package. ${ }^{94}$

\section{Results and Discussion}

A. Molecular Geometries. All molecules studied (Figure 1b) are derivatives of bis(benzyledene)piperidones and bis(benzyledene)cyclohexanones with different donor substituents $(R)$ in the para position on the side phenyl rings, and varying length of the $\pi$-bridges. Such $\mathrm{D}-\pi-\mathrm{A}-\pi-\mathrm{D}$ structures are typically associated with enhanced TPA properties. ${ }^{6,8,15,44}$ Experimentally characterized chromophores $\mathbf{2}-\mathbf{4}, \mathbf{6}-\mathbf{8}$, and $\mathbf{1 2}-\mathbf{1} 7^{58,59}$ have up to two unit $(n=2) \pi$-bridge length. We also consider structures 9-11 to evaluate the TPA enhancements in chromophores with longer $\pi$-bridges. Additionally, two different conformers are possible for molecules 1-11 with a methyl group of piperidone ring being in the equatorial or axial position. For example, these conformers for compound $\mathbf{7}$ are shown in Figure 2. We note that geometry optimization leads to the distortion from the 
planarity of equatorial structure. Single-crystal X-ray analysis shows "equatorial", "axial", and intermediate structures. 49,50,57,62,64 The energy difference between two conformations, estimated through B3LYP/6-31G** or HF/6-31G** calculations, does not exceed $3 \mathrm{~kJ} / \mathrm{mol}$, which may imply that in solution at roomtemperature one could find a statistical mixture of both conformers. Therefore, we study both "equatorial" and "axial" structures of compounds $\mathbf{5}-\mathbf{8}$ with strong TPA absorbancies to consider the impact of the conformational geometry on the optical properties of the chromophores.

As stated above, quantum-chemical optimizations of the ground-state geometries of chromophores at HF/6-31G level agree well with the experimental X-ray crystal data. $49,50,57,62,64$ Compared, for example, with optimizations based on the B3LYP functional, HF theory gives larger torsion angles between the plane of the central (pyperidone or cyclohexanone) ring and the side chains of the molecule, which is consistent with crystallographic data. Torsion angles (nonplanarity) of the smallest molecules with one pair of vinyl bridge segments (1$\mathbf{4 , 1 2 - 1 4 )}$ is much larger compared to torsional deformations of the molecules with longer $\pi$-bridges $(\mathbf{5}-\mathbf{1 1}, \mathbf{1 5}-\mathbf{1 7})$ reflecting steric distortions (for example $31.8^{\circ}(\mathrm{HF})$ and $22.9^{\circ}$ (B3LYP) for compound 3 and $4.3^{\circ}(\mathrm{HF})$ and $0.4^{\circ}(\mathrm{B} 3 \mathrm{LYP})$ for compound 7). The calculated electronic spectra of the chromophores under investigation are expected to be sensitive to several generic geometric parameters such as torsional distortions and bondlength alternation (BLA). The latter parameter reflects the degree of conjugation along the molecular backbone and can be defined as the difference in length between the single and double bonds $(r(\mathrm{C}-\mathrm{C})-r(\mathrm{C}=\mathrm{C}))$ for the vinyl bridges. ${ }^{95}$ For our compounds BLA was estimated to be about $0.14 \AA$ at the HF/6-31G level, which is consistent with our previous findings. ${ }^{79}$

B. Linear Absorption. The calculated absorption maxima and transition dipole moments are summarized in Table 1 and compared to experimental data when possible. ${ }^{58,59}$ Theory predicts two strong absorption bands corresponding to the excited states denoted as e in the visible region and $\mathrm{e}^{\prime}$ in the near UV, respectively. The two-peak spectra are observed in experiment as well, however, only the low energy peak was reported $^{58}$ and given in Table 1. TD-DFT based on the B3LYP hybrid kernel shows consistent agreement with experiment (see Table 1). Overall, calculations accurately reproduce the red shifts of the absorption maxima with increasing donor $(R)$ strength and elongation of the $\pi$ bridges. Compared to experiment, the vertical absorption maxima in the TD-DFT calculations are mostly blue shifted by $\sim 0.1-0.3 \mathrm{eV}$. This shift can be assigned to solute-solvent interactions, which typically reduce the excitation energies. Indeed, IEF-PCM calculations of chromophores $3,4,6,7,8,15,16$, and 17 in chloroform (see Table 1) red-shift excitation energies by $\sim 0.1-0.2 \mathrm{eV}$, which gives better agreement of experimental absorption maxima and theoretical transition frequencies. Compared to the gas-phase calculations, the solvent also slightly increases the transition dipole moments, which, in turn, leads to the larger TPA cross sections in solvent. Further improvement of the theoretical spectra may be obtained by taking into account vibrarional structure of electronic transitions based on the Franck-Condon analysis. ${ }^{96,97}$

Calculated linear absorption spectral line shapes for several compounds are shown in Figure 3. The intensity of the first peak (e) augments with an increase of the donor strength and elongation of the $\pi$ bridge. We observe near additivity of each vinyl unit contribution to the oscillator strength $\left(f \sim \mu_{\mathrm{ge}}{ }^{2}\right), \mu_{\mathrm{ge}}$ being the transition dipole moment. However, the second
TABLE 1: Linear Absorption Properties of Compounds $1-17^{a}$

\begin{tabular}{|c|c|c|c|c|c|}
\hline compound & $\begin{array}{c}\Omega_{\exp } \text { OPA } \\
\mathrm{eV}\end{array}$ & $\begin{array}{c}\Omega_{\mathrm{ge}}^{\mathrm{OPA}}, \\
\mathrm{eV}\end{array}$ & $\begin{array}{l}\mu_{\mathrm{ge}} \\
\mathrm{D}\end{array}$ & $\begin{array}{c}\Omega_{\mathrm{ge}^{\mathrm{e}}}{ }^{\mathrm{OPA}}, \\
\mathrm{eV}\end{array}$ & $\begin{array}{l}\mu_{\mathrm{ge}}{ }^{\prime}, \\
\mathrm{D}\end{array}$ \\
\hline $\mathbf{1}(\mathrm{eq})$ & - & $3.84(3)$ & 7.59 & $5.28(12)$ & 4.81 \\
\hline 2(eq) & - & $\begin{array}{l}3.34(2) \\
3.6(3)\end{array}$ & $\begin{array}{l}3.08 \\
7.78\end{array}$ & $5.15(11)$ & 3.32 \\
\hline $3(\mathrm{eq})$ & 2.65 & $3.05(1)$ & 9.29 & $4.62(9)$ & 6.39 \\
\hline $3 \mathrm{~s}(\mathrm{eq})$ & & $2.88(1)$ & 10.06 & $4.54(9)$ & 5.98 \\
\hline 4(eq) & 2.75 & $3.02(1)$ & 9.85 & $4.59(9)$ & 6.48 \\
\hline $4 s(e q)$ & & $2.86(1)$ & 10.54 & $4.51(7)$ & 3.37 \\
\hline $5(\mathrm{eq})$ & - & $3.19(3)$ & 11.05 & $4.25(6)$ & 7.73 \\
\hline $5(\mathrm{ax})$ & - & $3.16(2)$ & 10.97 & $4.24(6)$ & 7.62 \\
\hline $6(\mathrm{eq})$ & 3.00 & $2.99(2)$ & 10.45 & $4.1(6)$ & 7.82 \\
\hline $6 \mathrm{~s}(\mathrm{eq})$ & & $2.91(2)$ & 12.35 & $4.11(6)$ & 7.59 \\
\hline $6(a x)$ & 3.00 & $2.97(2)$ & 11.74 & $4.09(6)$ & 7.64 \\
\hline $6 \mathrm{~s}(\mathrm{ax})$ & & $2.91(2)$ & 12.35 & $3.59(5), 4.11(6)$ & 7.46 \\
\hline $7(\mathrm{eq})$ & 2.55 & $2.72(1)$ & 13.15 & $3.83(5)$ & 8.05 \\
\hline $7 \mathrm{~s}(\mathrm{eq})$ & & $2.56(1)$ & 13.55 & $3.76(5)$ & 8.16 \\
\hline $7(\mathrm{ax})$ & 2.55 & $2.7(2)$ & 13.17 & $3.85(6)$ & 7.35 \\
\hline $7 \mathrm{~s}(\mathrm{ax})$ & & $2.59(1)$ & 12.07 & $3.72(5)$ & 5.95 \\
\hline $8(\mathrm{eq})$ & 2.35 & $2.69(1)$ & 13.53 & $3.8(5)$ & 8.29 \\
\hline $8 \mathrm{~s}(\mathrm{eq})$ & & $2.53(1)$ & 13.96 & $3.73(5)$ & 8.35 \\
\hline $8(a x)$ & 2.35 & $2.68(1)$ & 13.65 & $3.81(5)$ & 7.83 \\
\hline $9(\mathrm{eq})$ & - & $2.85(2)$ & 13.4 & $3.77(6)$ & 9.76 \\
\hline $10(\mathrm{eq})$ & - & $2.47(1)$ & 15.59 & $3.40(5)$ & 10.24 \\
\hline 11(eq) & - & $2.63(2)$ & 15.78 & $3.43(6)$ & 11.36 \\
\hline 12 & - & $3.45(2)$ & 8.18 & $4.95(9)$ & 4.75 \\
\hline 13 & - & $3.11(1)$ & 8.1 & $4.56(8)$ & 5.47 \\
\hline 14 & - & $2.96(1)$ & 9.82 & $4.46(7)$ & 5.21 \\
\hline 15 & 3 & $2.96(1)$ & 12.12 & $4.05(4)$ & 7.8 \\
\hline $15 \mathrm{~s}$ & & $2.88(1)$ & 12.42 & $4.08(5)$ & 7.66 \\
\hline 16 & 2.55 & $2.69(1)$ & 13.25 & $3.79(4)$ & 8.28 \\
\hline $16 \mathrm{~s}$ & & $2.56(1)$ & 13.61 & $3.74(4)$ & 7.63 \\
\hline 17 & 2.52 & $2.66(1)$ & 13.63 & $3.77(4)$ & 8.41 \\
\hline $17 \mathrm{~s}$ & & $2.50(1)$ & 14.05 & $3.70(4)$ & 8.38 \\
\hline
\end{tabular}

${ }^{a}$ Experimental values of the absorption maxima $\Omega_{\exp }{ }^{\text {OPA }}$ are taken from ref 58. $\Omega_{\mathrm{ge}}{ }^{\mathrm{OPA}}$ and $\Omega_{\mathrm{ge}}{ }^{\mathrm{OPA}}$ are the calculated vertical absorption energies of the first (e) and second (e') optically allowed excited states with corresponding transition dipole moments $\mu_{\mathrm{ge}}$ and $\mu_{\mathrm{ge}}$. The letter "s" after the compound number refers to computational results obtained with the PCM solvation model. The respective excited-state numbers are given in the parentheses.

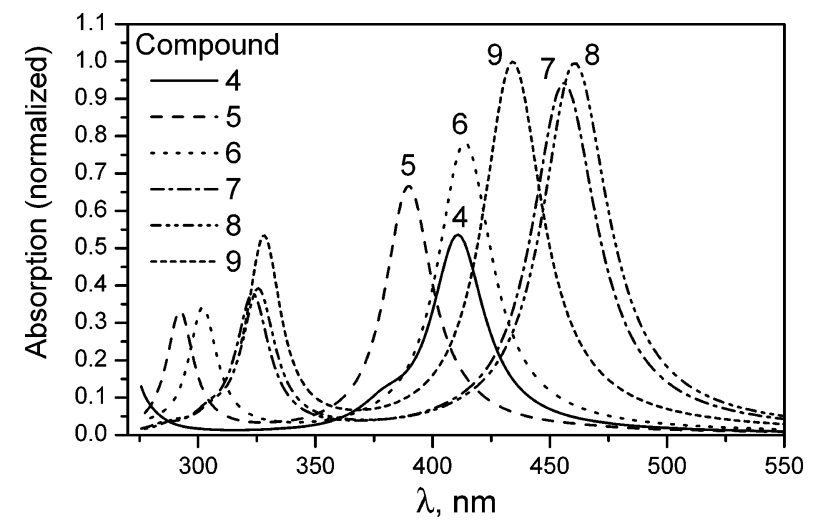

Figure 3. Calculated normalized linear absorption spectra of compounds 4-9. The OPA spectra are similar for the equatorial and axial conformations of the methyl group in the piperidone ring.

maximum ( $\mathrm{e}^{\prime}$ ) is not very sensitive to the substitution, but does grow with the $\pi$ bridge length. Calculated OPA parameters for axial and equatorial conformers of compounds 5-7 have no significant difference. Therefore, only spectra for equatorial conformations are shown in Figure 3. As expected, absorption spectra of piperidone and cyclohexanone derivatives are similar, since mostly carbonylic part of piperidone and cyclohexanone rings participates in the conjugation path contributing to the OPA. For most of the compounds (e.g., with dimethyl- and 


\begin{tabular}{|c|c|c|c|c|}
\hline \multicolumn{3}{|c|}{ Compound 10} & \multicolumn{2}{c|}{ Electron } \\
\hline $\begin{array}{c}\text { State } \\
S_{1}\end{array}$ \\
$\begin{array}{c}\text { OPA(e) } \\
99 \%\end{array}$
\end{tabular}

Figure 4. Natural transition orbitals describing the OPA and TPA states in compounds $\mathbf{1 0}$ and $\mathbf{1 1}$. Percentage indicates a fraction of the NTO pair contribution to a given electronic excitation.

diethylamino substituents), the lowest singlet state $\left(\mathrm{S}_{1}\right)$ is the most active state in the OPA spectra. In some compounds, however, the largest peak in the OPA spectra corresponds to the second $\left(\mathrm{S}_{2}\right)$ or even third $\left(\mathrm{S}_{3}\right)$ excited states (e.g., chromophores $\mathbf{1}, \mathbf{2}, \mathbf{5}, \mathbf{6}, \mathbf{7})$. In compound $\mathbf{7}$, solvent stabilizes the dipole-allowed OPA state back into the first excited state.

To analyze the nature of the excited states contributing to the linear absorption we show the relevant transition orbitals for selected compounds in Figures 2 and 4. The excited states for the considered family of chromophores with $C_{s}$ symmetry have $\mathrm{A}^{\prime}$ and $\mathrm{A}^{\prime \prime}$ symmetries. In our molecular family, the ground state $A^{\prime}$ is symmetric with respect to the mirror plane, and all OPA allowed states $A^{\prime \prime}$ are antisymmetric with respect to this plane. This partition has close analogy with $A_{g}$ and $B_{u}$ states in the centrosymmetric molecules, which are accordingly symmetric and antisymmetric with respect to the center of inversion. Electronic states e and $\mathrm{e}^{\prime}$ contributing to the OPA response of all molecules have a delocalized $\pi-\pi^{*}$ character. For example, transition orbitals of $S_{2}$ state (e) in chromophore 7 (Figure 2) show pronounced electronic density shifts to the conjugated center of the molecule: an electron is localized predominantly on the donor termini whereas a hole is extended over the core part of the chain. Wave functions of an electron and a hole have even and odd symmetries, respectively. This reflects the change of the wave function symmetry upon optical excitation to the state e. This transition can be well represented as a linear combination of neutral and zwitterionic basis resonance forms which is a typical case for push-pull chromophores. ${ }^{98}$ Similar description is applicable to the higher lying OPA state $\mathrm{e}^{\prime}$ (Figure 2). Here, wave functions of an electron and a hole have odd and even symmetries, respectively, and $\mathrm{e}^{\prime}$ has less zwitterionic contribution and, subsequently, less charge-transfer (CT) character compared to e. This may be the reason state $\mathrm{e}^{\prime}$ is not as sensitive to substitutions as state e in the OPA spectra (Figure 
3). The same description is valid for OPA states in all molecules 1-17. For example, relevant transition orbitals of chromophores $\mathbf{1 0}$ and 11 with longer $\pi$-bridges are shown in Figure 4. Even without donor substituents, state e in compound $\mathbf{1 1}$ has a weak CT trend toward the acceptor center of the molecule. We further note that all OPA states are not pure charge transfer ones (when an electron and a hole are well separated spatially), but rather states which undergo electron density redistribution from the donor to the acceptor, and thus have only a partial chargetransfer character.

In contrast, the lowest excited state $S_{1}$ in chromophore 7 (Figure 2) can be identified as a pure CT state with very small overlap between the electron (conjugated chain) and hole (piperidone ring) wave functions. This state has very weak transition dipole and always shows up as a $S_{1}$ or $S_{2}$ electronic transition in the computations of chromophores 1-12. It is wellknown and understood that TD-DFT based on pure and gradientcorrected functionals fails to describe CT states. ${ }^{99,100}$ This can be only partially corrected by hybrid functionals such as B3LYP. ${ }^{83,84}$ Most likely the low-lying CT states are not artifacts of TD-DFT and do exist in the compounds with piperidone ring 1-11. TD-HF calculations (not shown) predict this transition as well (though at higher energy). An important question, whether this CT transition affects molecular fluorescence by becoming the lowest state after the vibrational relaxation of the excited-state geometry, is a subject of future studies. Experimentally, molecules with cyclohexanone rings 12-17 show much stronger fluorescence compared to some of the compounds with piperidone rings, ${ }^{58}$ which indirectly supports the existence of low-lying CT states in 1-12.

C. Two-Photon Absorption. Calculated TPA properties of compounds 1-17 are summarized in Table 2. The second column of the table gives available experimental TPA cross sections. ${ }^{101,102}$ Direct comparison of theoretical results with experiment is not possible since spectroscopic measurements of the cross sections have been performed only for a narrow spectral range (850-900 nm), and therefore, they do not provide complete information on the TPA line shapes and peak maxima. Moreover, calculation have been done assuming a uniform empirical broadening parameter $\Gamma=0.1 \mathrm{eV}$ which is not the case for real molecular systems. ${ }^{81}$ Subsequently, this comparison can be used only as a guideline. Previously we observed an agreement between TD-DFT calculations and experimental data within $30 \%$ for TPA cross sections for similar organic compounds. ${ }^{79,81}$ Overall, the predicted TPA cross sections closely follow the experimental trends and agree within a factor of 2 for the absolute values. Similar to linear absorption, theory predicts two strong TPA absorption bands corresponding to the excited states denoted as E and $\mathrm{E}^{\prime}$. Typically the lowest TPA peak E corresponds to the fourth $\left(\mathrm{S}_{4}\right)$ or third $\left(\mathrm{S}_{3}\right)$ excited states and lies above the band-gap state e and CT states (see Table 2 ). The second TPA maximum $\mathrm{E}^{\prime}$ is attributed to a high-energy excited-state varying between $S_{11}$ (small molecules) and $S_{7}$ (large cyclohexanones) positions in the TD-DFT calculations.

In contrast to the large solvent stabilization of dipole-allowed OPA transitions (Table 1), the solvent only weakly affects the energies of TPA states due to their dipole-forbidden nature. IEF-PCM calculations of chromophores 4, 6-8, 15, 16, and 17 in chloroform (Table 2) red-shift excited state energies only by $\sim 0.05-0.1 \mathrm{eV}$. However, the TPA cross-section amplitudes increase notably by $\sim 10-30 \%$ when solvent effects are taken into account, which is consistent with our previous results. ${ }^{79,80}$ This can be rationalized by recalling the high sensitivity of two-
TABLE 2: Theoretical Two-Photon Absorption Properties of Compounds 1-17 and Available Experimental Values of TPA Cross Sections $\sigma_{\exp }{ }^{101} a$

\begin{tabular}{|c|c|c|c|c|c|}
\hline compound & $\begin{array}{l}\sigma_{\exp }, \\
\text { GM }\end{array}$ & $\begin{array}{c}\Omega_{\mathrm{gE}}^{\mathrm{TPA}}, \\
\mathrm{eV}\end{array}$ & $\begin{array}{c}\sigma_{\mathrm{gE}}^{\max }, \\
\mathrm{GM}\end{array}$ & $\begin{array}{c}\Omega_{\mathrm{gE}^{\mathrm{TPA}}}^{\mathrm{TPA}}, \\
\mathrm{eV}\end{array}$ & $\begin{array}{c}\sigma_{\mathrm{gE}^{\prime}}{ }^{\max }, \\
\mathrm{GM}\end{array}$ \\
\hline $\mathbf{1}(\mathrm{eq})$ & - & $4.12(4)$ & 88 & $5.28(12)$ & 102 \\
\hline 2(eq) & - & $3.84(4)$ & 125 & - & 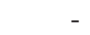 \\
\hline $3(\mathrm{eq})$ & $162 \pm 73$ & $3.38(4)$ & 298 & $4.8(10)$ & 223 \\
\hline $3 \mathrm{~s}(\mathrm{eq})$ & & $3.22(3)$ & 489 & $4.8(11)$ & 379 \\
\hline $4(\mathrm{eq})$ & $504 \pm 140$ & $3.36(4)$ & 291 & $4.76(10)$ & 244 \\
\hline $4 s(e q)$ & & $3.18(3)$ & 407 & $4.64(11)$ & 235 \\
\hline $5(\mathrm{eq})$ & - & $3.48(4)$ & 309 & $4.74(11)$ & 668 \\
\hline $5(\mathrm{ax})$ & - & $3.48(4)$ & 239 & $4.70(11)$ & 543 \\
\hline $6(\mathrm{eq})$ & $130 \pm 40$ & $3.32(3)$ & 562 & $4.40(9)$ & 531 \\
\hline $6 s(e q)$ & & $3.26(4)$ & 696 & $4.42(9)$ & 546 \\
\hline $6(\mathrm{ax})$ & $130 \pm 40$ & $3.30(4)$ & 363 & $4.36(8)$ & 382 \\
\hline $6 \mathrm{~s}(\mathrm{ax})$ & & $3.24(4)$ & 486 & $4.36(9)$ & 430 \\
\hline $7(\mathrm{eq})$ & $683 \pm 180$ & $3.02(3)$ & 788 & $4.18(9)$ & 522 \\
\hline $7 \mathrm{~s}(\mathrm{eq})$ & & $2.86(3)$ & 922 & $4.10(9)$ & 542 \\
\hline $7(\mathrm{ax})$ & $683 \pm 180$ & $3.00(3)$ & 1028 & 4.14(9) & 571 \\
\hline $7 \mathrm{~s}(\mathrm{ax})$ & & $2.94(3)$ & 1228 & $4.10(9)$ & 441 \\
\hline $8(\mathrm{eq})$ & $829 \pm 200$ & $3.00(3)$ & 1108 & $4.14(9)$ & 568 \\
\hline $8 \mathrm{~s}(\mathrm{eq})$ & & $2.84(3)$ & 1434 & $4.08(9)$ & 592 \\
\hline $8(a x)$ & $829 \pm 200$ & $2.96(3)$ & 1210 & $4.1(9)$ & 656 \\
\hline $9(\mathrm{eq})$ & - & $3.16(4)$ & 727 & $4.24(9)$ & 1312 \\
\hline $10(e q)$ & - & $2.74(2)$ & 2057 & $3.76(9)$ & 507 \\
\hline 11 & - & $2.90(3)$ & 1518 & $3.88(9)$ & 2776 \\
\hline 12 & - & $3.74(3)$ & 188 & $4.94(9)$ & 143 \\
\hline 13 & - & $3.38(3)$ & 295 & $4.66(10)$ & 62 \\
\hline 14 & - & $3.30(3)$ & 477 & $4.66(10)$ & 311 \\
\hline 15 & $135 \pm 30$ & $3.26(3)$ & 343 & $4.34(8)$ & 394 \\
\hline $15 \mathrm{~s}$ & & $3.22(3)$ & 667 & $4.30(8)$ & 618 \\
\hline 16 & $635 \pm 160$ & $2.96(2)$ & 981 & $4.08(7)$ & 590 \\
\hline $16 \mathrm{~s}$ & & $2.86(2)$ & 1314 & $4.00(7)$ & 571 \\
\hline 17 & $675 \pm 200$ & $2.94(2)$ & 1089 & $4.06(7)$ & 635 \\
\hline $17 \mathrm{~s}$ & & $2.80(2)$ & 1500 & $4.00(7)$ & 713 \\
\hline
\end{tabular}

${ }^{a} \Omega_{\mathrm{gE}}{ }^{\mathrm{TPA}}$ and $\Omega_{\mathrm{gE}}{ }^{\text {TPA }}$ are the calculated transition energies of the first $(\mathrm{E})$ and second $\left(\mathrm{E}^{\prime}\right)$ excited states active in TPA. The cross sections $\sigma_{\mathrm{gE}}{ }^{\max }$ and $\sigma_{\mathrm{gE}}{ }^{\max }$ are given for the maxima of the TPA peaks at approximately half of the corresponding transition energies. The letter "s" after the compound number refers to computational results obtained with the PCM solvation model. The excited-state numbers are given in the parentheses. $1 \mathrm{GM}=10^{-50} \mathrm{~cm}^{4} \mathrm{~s}_{\text {photon }}{ }^{-1}$.

photon absorbancies to the transition dipole moments between the relevant states. 6,8

Calculated TPA spectral line shapes for several compounds are shown in Figure 5. We first discuss comparison of TPA spectra of axial and equatorial conformers given in Figure 5a. Unlike the linear absorption, these conformers have different TPA cross-section maxima, while the absorption frequencies are similar. We note that for small molecules $(\mathbf{1}-\mathbf{6})$, the equatorial conformers have better TPA absorbancies compared to the axial ones. This trend is, however, reversed for larger compounds (e.g., 7-8). A possible rationalization of this observation is that the electronic excitations in the smaller molecules are in the strong confinement regime when positioning the lone pair in the molecular plane is beneficial for electronic delocalization, whereas the excited states of the larger compounds have better delocalization in a perfectly planar axial conformer. These subtleties of geometrical structure do not affect the linear absorption, but may show up in the nonlinear optical responses.

Variation of the TPA spectral line shapes with increase of the donor (R) strength and elongation of the $\pi$ bridges is illustrated in Figure 5b. Similar to the linear absorption, we observe significant red-shifts of the transition energies. The intensity of the first peak (E) grows with the increase of the donor strength and elongation of the $\pi$ bridge (see Figure 5b). Again, the second maximum ( $\left.\mathrm{E}^{\prime}\right)$ is not very sensitive to the 


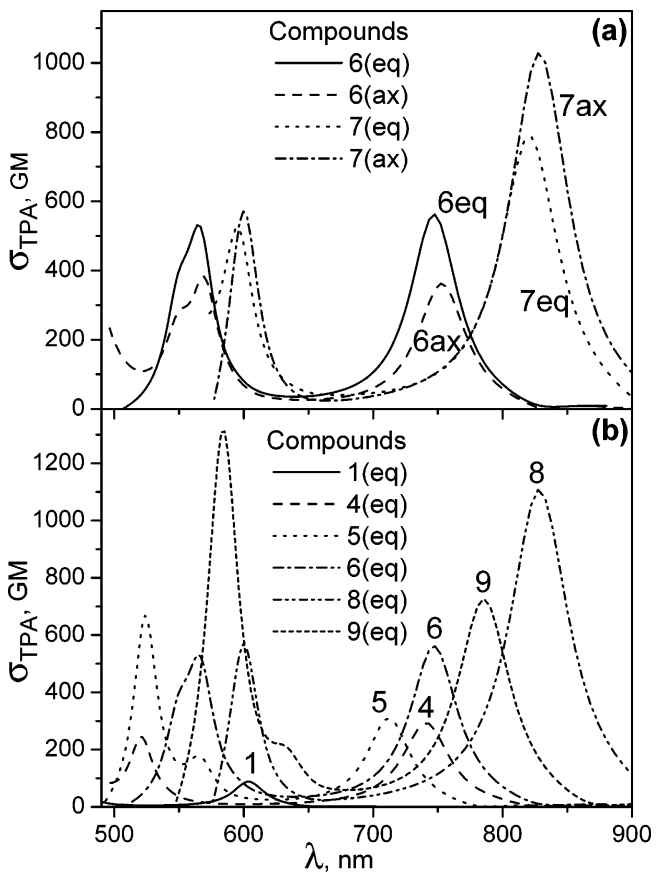

Figure 5. Calculated two-photon absorption spectra: (a) equatorial and axial conformations of 6 and 7, and (b) compounds $1,4,5,6,8$, and 9. $1 \mathrm{GM}=10^{-50} \mathrm{~cm}^{4} \mathrm{~s}_{\text {photon }}{ }^{-1}$.

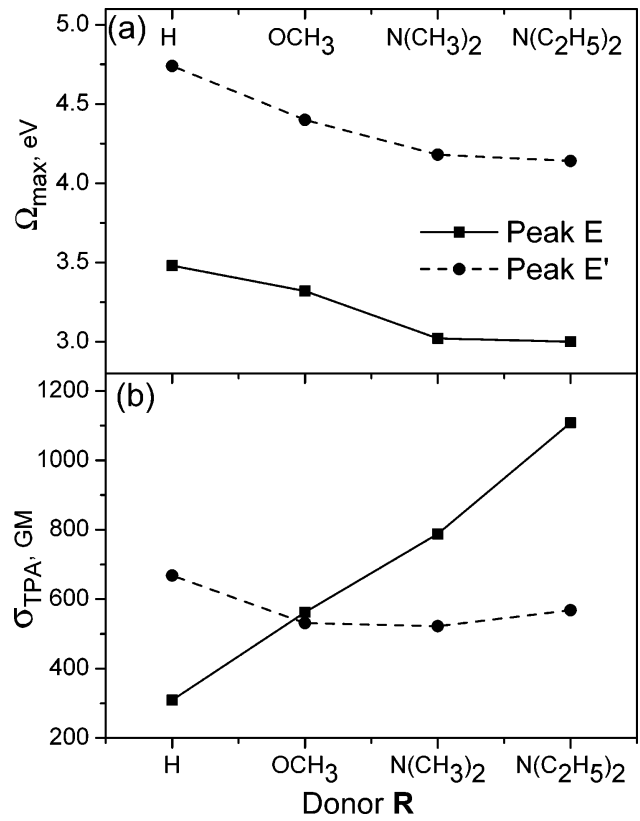

Figure 6. Variation of TPA properties with the strength of the terminal donor groups for compounds $\mathbf{5}-\mathbf{8}$. The lines connecting the points serve as a visual guide.

substitution (compare, e.g., 5, 6, and 8), but does grow with the $\pi$ bridge length (e.g., $\mathbf{5}$ vs $\mathbf{9}$ ). These trends of TPA activities are summarized in Figures 6 and 7. Energies of the excited states $\mathrm{E}$ and $\mathrm{E}^{\prime}$ are both shifted to the red by $\sim 0.5 \mathrm{eV}(\sim 0.25 \mathrm{eV}$ or 60-100 nm peak shifts of the respective TPA maxima) with an increase of the terminal donor strength (Figure 6a). The 4-fold enhancement of the TPA cross section with substitution is even more dramatic (Figure 6b). Changing the terminal group from methylamino to a somewhat stronger ethylamino donor substituent has a weak effect on the transition energies, but does increase the TPA cross sections. Figure 7a shows evolution of the TPA transition frequencies with the size of the $\pi$ bridge. We observe nearly perfect linear scaling of state energies plotted
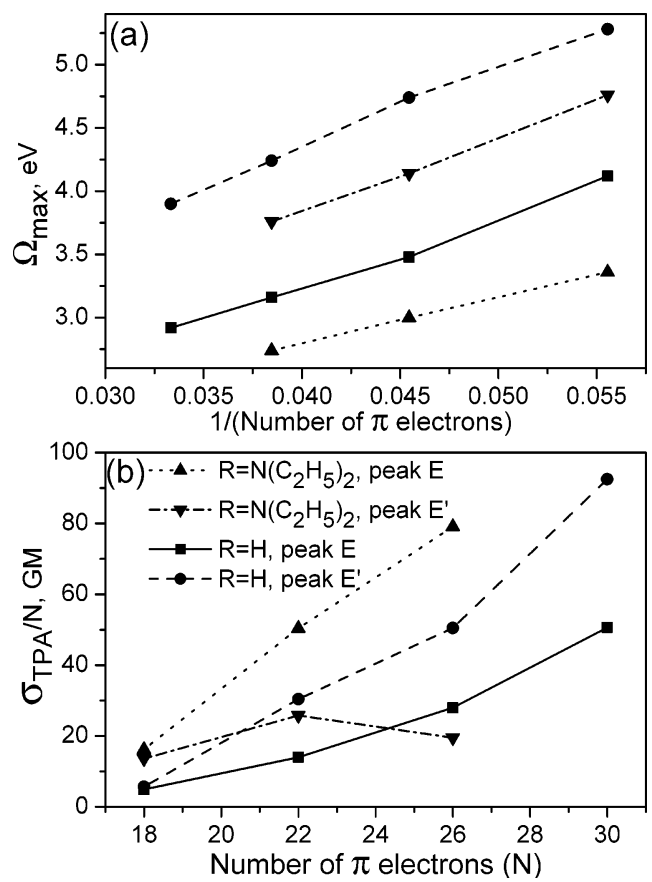

Figure 7. Variation of TPA properties with the number of $\pi$ electrons roughly representing the length of the $\pi$-bridge for compounds $\mathbf{1}, \mathbf{5}, \mathbf{9}$, and $11(\mathrm{R}=\mathrm{H})$ and 4,8 , and $10\left(\mathrm{R}=\mathrm{N}\left(\mathrm{C}_{2} \mathrm{H}_{5}\right)_{2}\right)$. The lines connecting the points serve as a visual guide.

as a function of the inverse number of $\pi$-electrons in the molecules. Such near-linear relationship is typical for bandgaps state in many conjugated polymers ${ }^{103-105}$ and is not completely unexpected. A remarkable observation is that this scaling law holds for both $\mathrm{E}$ and $\mathrm{E}^{\prime}$ peaks corresponding to the higher lying electronic states in molecules with and without substituents, and the slope of all curves is about the same. Dependence of the TPA amplitudes on the $\pi$ bridge length shown in Figure $7 \mathrm{~b}$ is not so straightforward. Intensities of both $\mathrm{E}$ and $\mathrm{E}^{\prime}$ peaks vary dramatically in unsubstituted molecules. Peak $\mathrm{E}^{\prime}$ is particularly sensitive to the elongation of the $\pi$ bridge. The situation is different for substituted chromophores. We observe a strong enhancement of $\mathrm{E}$, whereas amplitude of $\mathrm{E}^{\prime}$ is not affected by $\pi$ bridge length. The cyclohexanones compounds $(\mathbf{1 2}-\mathbf{1 7})$ exhibit the same trends as their piperidone counterparts $(\mathbf{2}-\mathbf{4}, \mathbf{6}-\mathbf{8})$ mediated by substitution and variation of $\pi$ bridge length. The TPA cross sections of large cyclohexanones are closer to that of axial piperidone conformers.

To compare quadrupolar molecular architectures with the dipolar ones, we investigated TPA properties of a modified compound $\mathbf{1 0}$ (Figure 1), with one of the two conjugated branches removed (not shown). Our calculations of the new molecule show that the excitation energies of TPA states increase by about $20 \%$, whereas the related transition dipoles decrease by $30-50 \%$ due to reduced conjugated chain length, compared to compound 10. Subsequently, TPA cross sections decrease by 3-4-fold. Observed trends can be well explained with simplified sum-over-states expression ${ }^{6,8}$ where the TPA cross section is proportional to the squared product of two transition dipoles over the excitation energy.

The transition orbitals shown in Figures 2 and 4 associated with TPA states $E$ and $E^{\prime}$ look remarkably similar to their OPA counterparts e and e', respectively. Since the TPA photoexcitation does not change the symmetry of the total electronic wave function, both electron and hole transition orbitals have even symmetries. However, the charge-transfer pattern discussed for the OPA transitions above, is applicable to the TPA states as 
well. This rationalizes the different effects produced by substitution and $\pi$ bridge length on the TPA amplitudes of the $\mathrm{E}$ and $\mathrm{E}^{\prime}$ maxima.

\section{Conclusion}

Design of functional chromophores for biological and medical applications involves optimization of many properties, such as molecular solubility in water and toxicity, in addition to photophysical response. The family of piperidone and cyclohexanone derivatives (Figure 1b) we considered show significant TPA activities, even though these are not the best cross sections among known organic chromophores. However, compounds 1-17 combine good nonlinear response with important cytotoxic properties $^{52-56,64}$ and may, therefore, serve as a synthetic base for future photodynamic therepy drugs.

Our study show that both OPA and TPA responses of these molecules can be well manipulated synthetically. Linear absorption of these compounds usually lies within 400-500 nm spectral window. Strong TPA absorbancies are calculated in $500-650$ and $700-900 \mathrm{~nm}$ wavelength regions. We found that OPA and TPA responses are dominated by two pairs of excited states. This gives rise the possibility of applying an effective five-state model (including the ground state) for the qualitative description of nonlinear optical responses in these chromophores, similar to the three-state approach used extensively for diaminobenzene and stilbene derivatives. ${ }^{6,8}$ Low (e and E) and high ( $\mathrm{e}^{\prime}$ and $\mathrm{E}^{\prime}$ ) energy electronic transitions show remarkably similar charge-transfer patterns, as evidenced by examination of the respective transition orbitals. The OPA and TPA peak positions can be tuned by either substitution with polar termini or elongation of the conjugated bridges (see Figures 3, 6a, and 7a). The TPA cross section of the low-frequency maximum can be significantly enhanced by donor substituents and an extension of the $\pi$ conjugation length (Figures $6 \mathrm{~b}$ and $7 \mathrm{~b}$ ). In contrast, substitution is not an effective approach to increase TPA absorbance at the lower wavelengths (peak E'). Here changing the $\pi$ bridge length remains an efficient method to achieve the desired responses (see Figure 7b). In organic solvents the OPA spectra show strong solvatochromic shifts to the red, while the intensities of the peaks are preserved. In contrast, we observe negligible solvatochromic shifts in the TPA spectra, while absorbancies increase up to $\sim 30 \%$. Our calculations show that even small changes in the molecular conformations ("equatorial" vs "axial" structures), which may not be reflected in the linear spectra, cause noticeable effects in the nonlinear spectra. These results provide theory-derived structure-properties relationships and capture a number of important trends in the linear and nonlinear optical responses of molecular family considered. This may further be used for synthetic design of new chromophores for future biomedical applications.

Acknowledgment. The research at LANL is supported by Center for Nonlinear Studies (CNLS) and the LDRD program of the U.S. Department of Energy. The research at NMHU is sponsored by NIH/RIMI Grant 1P20MD001104-01 and the NM EPSCoR program. This support is gratefully acknowledged. We are also grateful to Drs. S. S. Sarkisov, V. N. Nesterov, and R. Magyar for fruitful discussions.

\section{References and Notes}

(1) Göppert-Mayer, M. Ann. Phys. (Leipzig) 1931, 9, 273.

(2) Xu, C.; Zipfel, W.; Shear, J. B.; Williams, R. M.; Webb, W. W. Proc. Natl. Acad. Sci. U.S.A. 1996, 93, 10763.

(3) Heikal, A. A.; Huang, S. H.; Halik, M.; Marder, S. R.; Wenseleers, W.; Perry, J. W.; Webb, W. W. Biophys. J. 2002, 82, 493A.
(4) Reinhardt, B. A.; Brott, L. L.; Clarson, S. J.; Dillard, A. G.; Bhatt, J. C.; Kannan, R.; Yuan, L. X.; He, G. S.; Prasad, P. N. Chem. Mater. $1998,10,1863$.

(5) Baur, J. W.; Alexander, M. D.; Banach, M.; Denny, L. R.; Reinhardt, B. A.; Vaia, R. A.; Fleitz, P. A.; Kirkpatrick, S. M. Chem. Mater. 1999, 11, 2899.

(6) Albota, M.; Beljonne, D.; Brédas, J. L.; Ehrlich, J. E.; Fu, J. Y. Heikal, A. A.; Hess, S. E.; Kogej, T.; Levin, M. D.; Marder, S. R.; McCord Maughon, D.; Perry, J. W.; Rockel, H.; Rumi, M.; Subramaniam, C.; Webb, W. W.; Wu, X. L.; Xu, C. Science 1998, 281, 1653.

(7) Rumi, M.; Ehrlich, J. E.; Heikal, A. A.; Perry, J. W.; Barlow, S.; Hu, Z. Y.; McCord-Maughon, D.; Parker, T. C.; Rockel, H.; Thayumanavan, S.; Marder, S. R.; Beljonne, D.; Bredas, J. L. J. Am. Chem. Soc. 2000, 122, 9500 .

(8) Pond, S. J. K.; Rumi, M.; Levin, M. D.; Parker, T. C.; Beljonne, D.; Day, M. W.; Bredas, J. L.; Marder, S. R.; Perry, J. W. J. Phys. Chem. A 2002, 106, 11470 .

(9) Werts, M. H. V.; Gmouh, S.; Mongin, O.; Pons, T.; BlanchardDesce, M. J. Am. Chem. Soc. 2004, 126, 16294.

(10) Porres, L.; Mongin, O.; Katan, C.; Charlot, M.; Pons, T.; Mertz, J.; Blanchard-Desce, M. Org. Lett. 2004, 6, 47.

(11) Ventelon, L.; Moreaux, L.; Mertz, J.; Blanchard-Desce, M. Synth. Met. 2002, 127, 17 .

(12) Goodson, T., III. Annu. Rev. Phys. Chem. 2005, 56, 581.

(13) Goodson, T. G. Acc. Chem. Res. 2005, 38, 99.

(14) Bhawalkar, J. D.; He, G. S.; Prasad, P. N. Rep. Prog. Phys. 1996, 59,1041

(15) Lin, T. C.; Chung, S. J.; Kim, K. S.; Wang, X. P.; He, G. S.; Swiatkiewicz, J.; Pudavar, H. E.; Prasad, P. N. Polym. Photonics Appl. II 2003, 161, 157

(16) Joshi, M. P.; Pudavar, H. E.; Swiatkiewicz, J.; Prasad, P. N.; Reianhardt, B. A. Appl. Phys. Lett. 1999, 74, 170

(17) Denk, W.; Strickler, J. H.; Webb, W. W. Science 1990, 248, 73.

(18) Cianci, G. C.; Wu, J. R.; Berland, K. M. Microsc. Res. Tech. 2004, 64, 135 .

(19) McConnell, G.; Gu, E.; Griffin, C.; Jeon, C. W.; Choi, H. W.; Gurney, A. M.; Girkin, J. M.; Dawson, M. D. Des. Nat. 2004, 6, 341.

(20) Palmer, G. M.; Keely, P. J.; Breslin, T. M.; Ramanujam, N. Photochem. Photobiol. 2003, 78, 462.

(21) Kuzmin, A. N.; Kachynski, A. V.; Ohulchanskyy, T. Y.; Roy, I.; Prasad, P. N.; Bruckenstein, S. Appl. Phys. Lett. 2004, 84, 2454.

(22) Cumpston, B. H.; Ananthavel, S. P.; Barlow, S.; Dyer, D. L.; Ehrlich, J. E.; Erskine, L. L.; Heikal, A. A.; Kuebler, S. M.; Lee, I. Y. S.; McCord-Maughon, D.; Qin, J. Q.; Rockel, H.; Rumi, M.; Wu, X. L.; Marder, S. R.; Perry, J. W. Nature (London) 1999, 398, 51.

(23) Shen, Y. Z.; Swiatkiewicz, J.; Jakubczyk, D.; Xu, F. M.; Prasad, P. N.; Vaia, R. A.; Reinhardt, B. A. Appl. Opt. 2001, 40, 938.

(24) Pudavar, H. E.; Joshi, M. P.; Prasad, P. N.; Reinhardt, B. A. Appl. Phys. Lett. 1999, 74, 1338 .

(25) Kawata, S.; Kawata, Y. Chem. Rev. 2000, 100, 1777.

(26) Spangler, C. W. J. Mater. Chem. 1999, 9, 2013.

(27) Ehrlich, J. E.; Wu, X. L.; Lee, I. Y. S.; Hu, Z. Y.; Rockel, H.; Marder, S. R.; Perry, J. W. Opt. Lett. 1997, 22, 1843.

(28) Bauer, C.; Schnabel, B.; Kley, E. B.; Scherf, U.; Giessen, H.; Mahrt, R. F. Adv. Mater. 2002, 14, 673 .

(29) He, G. S.; Signorini, R.; Prasad, P. N. Appl. Opt. 1998, 37, 5720.

(30) Konig, K. J. Microsc. 2000, 200, 83.

(31) Zipfel, W. R.; Williams, R. M.; Christie, R.; Nikitin, A. Y.; Hyman,

B. T.; Webb, W. W. Proc. Natl. Acad. Sci. U.S.A. 2003, 100, 7075.

(32) Blanchard-Desce, M. C. R. Acad. Sci., Ser. IV (Phys., Astrophys.) 2002, 3, 439 .

(33) Kohler, R. H.; Cao, J.; Zipfel, W. R.; Webb, W. W.; Hanson, M. R. Science 1997, 276, 2039.

(34) Miller, M. J.; Wei, S. H.; Parker, I.; Cahalan, M. D. Science 2002, 296,1869

(35) Fisher, A. M. R.; Murphree, A. L.; Gomer, C. J. Lasers Surg. Med.

$1995,17,2$

(36) Bhawalkar, J. D.; Kumar, N. D.; Zhao, C. F.; Prasad, P. N. J. Clin. Laser Med. Surg. 1997, 15, 201

(37) Pettit, D. L.; Wang, S. S. H.; Gee, K. R.; Augustine, G. J. Neuron 1997, 19, 465.

(38) Prasad, P. N. Introduction to Biophotonics; Wiley-Interscience: New York, 2003.

(39) Prasad, P. N. Nanophotonics; Wiley-Interscience: New York, 2004

(40) Weishaupt, K. R.; Dougherty, T. J. Radiat. Res. 1978, 74, 586.

(41) Dougherty, T. J.; Kaufman, J. E.; Goldfarb, A.; Weishaupt, K. R.; Boyle, D.; Mittleman, A. Cancer Res. 1978, 38, 2628.

(42) Dougherty, T. J. J. Natl. Cancer Inst. 1974, 52, 1333. 481 .

(44) Hales, J. M.; Hagan, D. J.; Stryland, E. W. V.; Schafer, K. J.; Morales, A. R.; Belfield, K. D.; Pacher, P.; Kwon, O.; Zojer, E.; Bredas, J. L. J. Chem. Phys. 2004, 121, 3152. 
(45) Katan, C.; Terenziani, F.; Mongin, O.; Werts, M. H. V.; Porres, L.; Pons, T.; Mertz, J.; Tretiak, S.; Blanchard-Desce, M. J. Phys. Chem. A 2005, 109, 3024.

(46) Kawamata, J.; Inoue, K.; Kasatani, H.; Terauchi, H. Jpn. J. Appl. Phys. 1992, 31, 254.

(47) Kawamata, J.; Inoue, K. Chem. Lett. 1993, 6, 921.

(48) Kawamata, J.; Inoue, K.; Inabe, T. Mol. Cryst. Liq. Cryst. 1996 278-279, 117.

(49) Yakimanski, A. V.; Kolb, U.; Matveeva, G. N.; Voigt-Martin, I. G.; Tenkovtsev, A. V. Acta Crystallogr. A: Found. Crystallogr. 1997, A53, 603.

(50) Kawamata, J.; Inoue, K.; Inabe, T. Bull. Chem. Soc. Jpn. 1998, $71,2777$.

(51) Kawamata, J.; Akiba, M.; Inagaki, Y. Jpn. J. Appl. Phys. 2003, 42, L17

(52) Dimmock, J. R.; Arora, V. K.; Quail, J. W.; Pugazhenthi, U.; Allen, T. M.; Kao, G. Y.; Clercq, E. D. J. Pharm. Sci. 1994, 83, 1124.

(53) Dimmock, J. R.; Vashishtha, S. C.; Quail, J. W.; Pugazhenthi, U.; Zimpel, Z.; Sudom, A. M.; Allen, T. M.; Kao, G. Y.; Balzarini, J.; Clercq, E. D. J. Med. Chem. 1998, 41, 4012.

(54) Dimmock, J. R.; Zello, G. A.; Oloo, E. O.; Quail, J. W.; Kraatz, H. B.; Perjesi, P.; Aradi, F.; Takacs-Novak, K.; Allen, T. M.; Santos, C. L.; Balzarini, J.; Clercq, E. D.; Stables, J. P. J. Med. Chem. 2002, 45, 3103.

(55) Dimmock, J. R.; Kandepu, N. M.; Nazarali, A. J.; Motaganahalli, N. L.; Kowalchuk, T. P.; Pugazhenthi, U.; Prisciak, J. S.; Quail, J. W.; Allen, T. M.; LeClerc, R.; Santos, C. L.; Clercq, E. D.; Balzarini, J. J. Med. Chem. 2000, 43, 3933.

(56) Dimmock, J. R.; Padmanilayam, M. P.; Puthucode, R. N.; Nazarali, A. J.; Motaganahalli, N. L.; Zello, G. A.; Quail, J. W.; Oloo, E. O.; Kraatz, H. B.; Prisciak, J. S.; Allen, T. M.; Santos, C. L.; Balzarini, J.; Clercq, E. D.; Manavathu, E. K. J. Med. Chem. 2001, 44, 586.

(57) Nesterov, V. N.; Timofeeva, T. V.; Sarkisov, S. S.; Leyderman, A.; Lee, C. Y.; Antipin, M. Y. Acta Crystallogr. C: Cryst. Str. Comm. 2003, 59, o605.

(58) Peterson, B. H.; Sarkisov, S. S.; Nesterov, V. N.; Radovanova, E. I.; Leyderman, A.; Timofeeva, T.; Antipin, M.; Curley, M. J.; Fleitz, P. A.; Lee, C.; Wang, J. C. Proc. SPIE: Int. Soc. Opt. Eng. 2004, 5323, 365.

(59) Sarkisov, S. S.; Peterson, B. H.; Curley, M. J.; Nesterov, V. N. Timofeeva, T.; Antipin, M.; Radovanova, E. I.; Leyderman, A.; Fleitz, P. A. J. Nonlinear Opt. Phys. Mater. 2005, 14, 21.

(60) Szakacs, G.; Annereau, J. P.; Lababidi, S.; Shankavaram, U. Arciello, A.; Bussey, K. J.; Reinhold, W.; Guo, Y. P.; Kruh, G. D.; Reimers, M.; Weinstein, J. N.; Gottesman, M. M. Cancer Cell 2004, 6, 129.

(61) Jia, Z. C.; Quail, J. W.; Arora, V. K.; Dimmock, J. R. Acta Crystallogr. C: Cryst. Struct. Commun. 1989, 45, 1117.

(62) Jia, Z. C.; Quail, J. W.; Arora, V. K.; Dimmock, J. R. Acta Crystallogr. C: Cryst. Struct. Commun. 1989, 45, 285.

(63) Jia, Z. C.; Quail, J. W.; Arora, V. K.; Dimmock, J. R. Acta Crystallogr. C: Cryst. Struct. Commun. 1988, 44, 2114.

(64) Dimmock, J. R.; Jha, A.; Zello, G. A.; Quail, J. W.; Oloo, E. O.; Nienaber, K. H.; Kowalczyk, E. S.; Allen, T. M.; Santos, C. L.; Clercq, E. D.; Balzarini, J.; Manavathu, E. K.; Stables, J. P. Eur. J. Med. Chem. 2002, 37, 961.

(65) Nesterov, V. N. Acta Crystallogr. C: Cryst. Struct. Commun. 2004, 60, o806.

(66) Akiba, M.; Morinaga, N.; Takizawa, H.; Ogiyama, M.; Ichijima, S.; Tani, T.; Harada, A.; Yanagaku, Y. Presented at Nonlinear Optical Properties of Organic Molecules and Crystals, 2005. Abstract ICOPE2005/ ICONO' 8, p 227.

(67) Tretiak, S.; Mukamel, S. Chem. Rev. 2002, 102, 3171.

(68) Stanton, J. F. J. Chem. Phys. 2001, 115, 10382.

(69) Szabo, A.; Ostlund, N. S. Modern Quantum Chemistry: Introduction to Advanced Electronic Structure Theory; McGraw-Hill: New York, 1989.

(70) Dewar, M. J. S.; Zoebisch, E. G.; Healy, E. F.; Stewart, J. J. P. J. Am. Chem. Soc. 1985, 107, 3902.

(71) Ridley, J.; Zerner, M. C. Theor. Chim. Acta 1973, 32, 111.

(72) Runge, E.; Gross, E. K. U. Phys. Rev. Lett. 1984, 52, 997.

(73) Casida, M. E. In Recent Advances in Density-Functional Methods; Chong, D. A., Ed.; World Scientific: Singapore, 1995; Vol. 3 of Part 1.
(74) Casida, M. E.; Jamorski, C.; Casida, K. C.; Salahub, D. R. J. Chem. Phys. 1998, 108, 4439.

(75) Furche, F.; Ahlrichs, R. J. Chem. Phys. 2002, 117, 7433.

(76) Salek, P.; Vahtras, O.; Guo, J. D.; Luo, Y.; Helgaker, T.; Agren, H. Chem. Phys. Lett. 2003, 374, 446.

(77) Berman, O.; Mukamel, S. Phys. Rev. A 2003, 67, 42503.

(78) Tretiak, S.; Chernyak, V. J. Chem. Phys. 2003, 119, 8809.

(79) Masunov, A. M.; Tretiak, S. J. Phys. Chem. B 2004, 108, 899.

(80) Kobko, N.; Masunov, A.; Tretiak, S. Chem. Phys. Lett. 2004, 392, 444.

(81) Bartholomew, G. P.; Rumi, M.; Pond, S. J. K.; Perry, J. W.; Tretiak, S.; Bazan, G. C. J. Am. Chem. Soc. 2004, 126, 11529.

(82) Frisch, M. J.; Trucks, G. W.; Schlegel, H. B.; Scuseria, G. E.; Robb, M. A.; Cheeseman, J. R.; Zakrzewski, V. G.; Montgomery, J. A., Jr.; Stratmann, R. E.; Burant, J. C.; Dapprich, S.; Millam, J. M.; Daniels, A. D.; Kudin, K. N.; Strain, M. C.; Farkas, O.; Tomasi, J.; Barone, V.; Cossi, M.; Cammi, R.; Mennucci, B.; Pomelli, C.; Adamo, C.; Clifford, S.; Ochterski, J.; Petersson, G. A.; Ayala, P. Y.; Cui, Q.; Morokuma, K.; Malick, D. K.; Rabuck, A. D.; Raghavachari, K.; Foresman, J. B.; Cioslowski, J.; Ortiz, J. V.; Baboul, A. G.; Stefanov, B. B.; Liu, G.; Liashenko, A.; Piskorz, P.; Komaromi, I.; Gomperts, R.; Martin, R. L.; Fox, D. J.; Keith, T.; AlLaham, M. A.; Peng, C. Y.; Nanayakkara, A.; Challacombe, M.; Gill, P. M. W.; Johnson, B.; Chen, W.; Wong, M. W.; Andres, J. L.; Gonzalez, C.; Head-Gordon, M.; Replogle, E. S.; Pople, J. A. Gaussian 98 (Revision A.11); Gaussian, Inc.: Pittsburgh, PA, 2002.

(83) Becke, A. D. J. Chem. Phys. 1993, 98, 5648.

(84) Becke, A. D. J. Chem. Phys. 1993, 98, 1372.

(85) Mukamel, S. Principles of Nonlinear Optical Spectroscopy; Oxford: New York, 1995.

(86) Das, G. P.; Yeates, A. T.; Dudis, D. S. Chem. Phys. Lett. 2002, 361,71 .

(87) Zhou, X.; Ren, A. M.; Feng, J. K.; Liu, X. J. J. Phys. Chem. A 2003, 107,1850

(88) Garito, A. F.; Heflin, J. R.; Wong, K. Y.; Zamani-Khamiri, O. In Organic Materials for Nonlinear Optics; Hann, R. A., Bloor, D., Eds.; Royal Chemical Society: Burlington House, New York, 1989).

(89) Cances, E.; Mennucci, B.; Tomasi, J. J. Chem. Phys. 1997, 107, 3032.

(90) Cossi, M.; Barone, V.; Mennucci, B.; Tomasi, J. Chem. Phys. Lett. 1998, 286, 253.

(91) Mennucci, B.; Cammi, R.; Tomasi, J. J. Chem. Phys. 1998, 109, 2798.

(92) Cossi, M.; Barone, V. J. Chem. Phys. 2001, 115, 4708.

(93) Martin, R. L. J. Chem. Phys. 2003, 118, 4775.

(94) Kokalj, A. J. Mol. Graph. Model. 1999, 17, 176.

(95) Marder, S. R.; Perry, J. W.; Tiemann, B. G.; Gorman, C. B.; Gilmour, S.; Biddle, S. L.; Bourhill, G. J. Am. Chem. Soc. 1993, 115, 2524 (96) Dierksen, M.; Grimme, S. J. Phys. Chem. A 2004, 108, 10225.

(97) Magyar, R. J.; Tretiak, S.; Gao, Y.; Wang, H. L.; Shreve, A. P. Chem. Phys. Lett. 2005, 401, 149.

(98) Moran, A. M.; Kelley, A. M.; Tretiak, S. Chem. Phys. Lett. 2003, 367, 293.

(99) Tozer, D. J. J. Chem. Phys. 2003, 119, 12697.

(100) Dreuw, A.; Head-Gordon, M. J. Am. Chem. Soc. 2004, 126, 4007.

(101) Experimental TPA cross sections of compounds 1-17 reported in ref 58 are overestimated. We are giving renormalized experimental values of TPA cross sections calibrated against Rhodamine $\mathrm{B}^{43}$ and reported in ref 59 .

(102) Peterson, B. H.; Sarkisov, S. S.; Nesterov, V. N.; Radovanova, E. I.; Leyderman, A.; Timofeeva, T.; Antipin, M.; Curley, M. J.; Fleitz, P. A.; Lee, C.; Wang, J.-C. Proc. SPIE: Int. Soc. Opt. Eng. 2004, 5351, 181.

(103) Woo, H. S.; Lhost, O.; Graham, S. C.; Bradley, D. D. C.; Friend, R. H.; Quattrocchi, C.; Bredas, J. L.; Schenk, R.; Mullen, K. Synth. Met. 1993, 59, 13 .

(104) Tretiak, S.; Chernyak, V.; Mukamel, S. Phys. Rev. Lett. 1996 77,4656 .

(105) Hutchison, G. R.; Zhao, Y. J.; Delley, B.; Freeman, A. J.; Ratner, M. A.; Marks, T. J. Phys. Rev. B 2003, 68, 035204. 